\title{
Freqüência e percentual de suscetibilidade de bactérias isoladas em pacientes atendidos na Unidade de Terapia Intensiva do Hospital Geral de Fortaleza
}

\author{
Frequency and susceptibility percentile of bacteria isolated in patients assisted in the Intensive \\ Care Unit of the General Hospital of Fortaleza
}

Everardo Albuquerque Menezes'; Kélvia Miranda Sá'; Francisco Afrânio Cunha'; Maria Rozellê Ferreira Ângelo²; Inácio Regis Nascimento Oliveiraz; Maria Núbia Cavalcante Salviano²

\section{unitermos resumo}

Infecçōes hospitalares

Introdução: As infecções hospitalares, hoje, são motivo de grande preocupação no âmbito hospitalar, principalmente nas unidades de terapia intensiva (UTIs), nas quais encontramos uma associação de fatores propícios ao surgimento de infecções. Objetivos: Verificar a freqüência e o perfil de suscetibilidade aos antimicrobianos das bactérias isoladas de pacientes da UTI do Hospital Geral de Fortaleza (HGF). Material

Resistência bacteriana e métodos: As bactérias foram isoladas em meio de cultura e a identificação e o teste de suscetibilidade aos antimicrobianos realizados por meio do sistema de automação MicroScan WalkWay. Resultados: No período de janeiro a dezembro de 2002 houve $34 \%$ de positividade de bactérias no aspirado traqueal de pacientes da UTI; $10 \%$ de positividade no cateter; $26 \%$ de positividade na urina; e $30 \%$ de positividade no sangue. As bactérias mais freqüentes do aspirado traqueal foram Pseudomonas aeruginosa (16\%) e Klebsiella pneumoniae (15\%). Em cateteres, houve maior freqüência de Staphylococcus coagulase negativa (SCN) (25\%) e Staphylococcus aureus (25\%); na urina, predominaram Klebsiella pneumoniae (16\%) e Pseudomonas aeruginosa (14\%). Em hemoculturas, as bactérias mais isoladas foram SCN (41\%) e Staphylococcus aureus (17\%). Foi observado, em relação ao perfil de suscetibilidade, que as Pseudomonas aeruginosa isoladas de aspirado traqueal apresentaram total suscetibilidade à piperacilina e resistência total à ceftriaxona e à cefotaxima. A Klebsiella pneumoniae isolada de aspirados traqueais foi totalmente sensível ao imipenem, não apresentando resistência total a nenhum antimicrobiano testado, e apresentou suscetibilidade de $54 \%$ à ceftazidima. Em cateter ela apresentou resistência a ampicilina/sulbactam, cefepima, cefotaxima, ceftazidima, ceftriaxona, cefuroxima, gentamicina, piperacilina/tazobactam, piperacilina, ticarcilina/clavulanato e tobramicina, bem como suscetibilidade ao imipenem. Na urina, observamos $55 \%$ de suscetibilidade para ciprofloxacina. Os S. aureus e SCN foram isolados principalmente do cateter, sendo suscetíveis à vancomicina (100\%). Conclusão: Os patógenos que mais causaram infecções na UTI do HGF foram Pseudomonas aeruginosa, Klebsiella pneumoniae, Acinetobacter baumannii, S aureus e SCN.
\end{abstract}

abstract

Introduction: Nosocomial infections are prominent problem in hospital environment, mainly in intensive care units (ICU), where innumerous factors favoring the development of these infections are found. Objectives: To determine the frequency and the antibiotic resistance pattern of bacteria isolated from ICU patients in the General Hospital of Fortaleza (HGF). Material and methods: Bacteria were isolated in culture medium and the identification and test of susceptibility to antimicrobials was performed using MicroScan WalkWay automation device. Results: From January to December of 2002, 34\% of specimens from tracheal secretion; $10 \%$ from catheter cultures; $26 \%$ from urine and 30\% from the blood yielded isolates. Specimens more frequent in tracheal secretion were Pseudomonas aeruginosa (16\%) e Klebsiella pneumoniae (15\%). In catheter cultures, we found high prevalence of Staphylococcus negative coagulase (SNC) (25\%) and Staphylococcus aureus (25\%); in urine, Klebsiella pneumoniae (16\%) and Pseudomonas aeruginosa (14\%) were the most prevalent. From blood, we isolated mostly SNC (41\%) and Staphylococcus aureus (17\%). About antimicrobial susceptibility patterns of Pseudomonas aeruginosa isolated from tracheal secretion, we found a high sensitivity to piperacilin and high resistance to ceftriaxone and cefotaxime. Klebsiella pneumoniae isolated fom tracheal secretion showed high sensibility to imipenem, but no resistance to other antimicrobials althogeter. Susceptibility to ceftazidime was $54 \%$. Isolates from catheters showed broad resistance pattern (ampicillin/sulbactam, cefepime, cefotaxime, ceftazidime, ceftriaxone, cefuroxime, gentamicin, piperacillin/tazobactam, piperacillin, ticarcillin/clavulani acid and tobramycin) but imipenem susceptibility. In urine, the susceptibility to ciprofloxacin was 55\%. Staphylococcus aureus e SNC were isolated mainly from catheter, with high susceptibility to vancomycin (100\%). Discussion: Antibiotic resistance is an increasing problem challenging ICUs worldwide. Infections due to multiresistant bacteria correlate with increased morbidity and mortality in severely ill patients. Conclusion: The most important pathogens causing infections in ICU of HGF were P. aeruginosa. K. pneumoniae, Acinetobacter baumannii, S. aureus and SNC. key words Nosocomial infections

Antimicrobial resistance

Intensive care unit 


\section{Introdução}

A infecção hospitalar pode ser definida como qualquer infecção adquirida após a internação do paciente, que se manifesta durante a internação ou mesmo após a alta, e que pode ser relacionada com a internação e/ou procedimentos hospitalares ${ }^{(8,24)}$. Essa infecção pode ser causada pela microbiota do próprio paciente, ou por microrganismos encontrados no ambiente em que ele vivia. Em nosso país, a incidência de infecções hospitalares (ou adquiridas nos hospitais) é maior nos hospitais universitários ou de ensino do que nos demais hospitais da comunidade. Esse aumento é atribuído à maior gravidade das doenças e/ou aos casos ou procedimentos mais complicados realizados nos hospitais de ensino. Internações hospitalares mais longas e interação mais efetiva dos pacientes com vários profissionais da área de saúde, além de estudantes e membros da equipe, contribuem para esse aumento ${ }^{(3,24)}$.

Nesse ambiente, o uso racional de antimicrobianos torna-se arma essencial do sistema de prevenção de infecções nosocomiais. A literatura tem mostrado que o sistema de auditoria dos antimicrobianos prescritos por equipe multidisciplinar dedicada a essa função é a forma mais eficaz de racionalizar esse uso, passando então a ser ferramenta fundamental do sistema preventivo(4).

O conhecimento dos princípios gerais que norteiam o uso de antimicrobianos, assim como das propriedades e características básicas dos antimicrobianos disponíveis, permitiu estabelecer critérios científicos que dão segurança à sua indicação terapêutica e/ou profilática. Vale lembrar que o aumento do espectro de atividade antimicrobiana nem sempre representa vantagem, visto que, quando muito amplo, pode propiciar o aparecimento de superinfecção(9).

Para seleção do(s) antimicrobiano(s) a ser(em) prescrito(s), sempre devem ser considerados três aspectos fundamentais: o estado clínico do paciente, o local ou sítio da infecção e o agente etiológico presumido ou comprovado ${ }^{(18)}$.

Portanto, tendo em vista o grande número de antimicrobianos disponíveis, a complexidade de determinados tipos de infecção, especialmente as que acometem pessoas imunodeprimidas, e os diversos mecanismos de resistência apresentados pelos microrganismos, passou também a ser mais complexa a correta avaliação da sensibilidade aos agentes antimicrobianos ${ }^{(2,14,15,18)}$.

Vários fatores modulam a interação entre $o$ antimicrobiano, o microrganismo e o paciente, sendo muitas vezes indispensável a análise conjunta da situação por parte do médico e do microbiologista para que a conduta terapêutica a ser adotada seja a mais correta possível ${ }^{(1,13)}$.

Pelo que foi exposto, e em razão da grande importância da evolução da resistência microbiana e da necessidade de um controle das infecções hospitalares em áreas de maior risco, como as unidades de terapia intensiva (UTIs), é que surgiu o interesse de se fazer um estudo da freqüência de bactérias na UTI do Hospital Geral de Fortaleza (HGF), analisando também a suscetibilidade desses microrganismos frente aos antimicrobianos utilizados na UTI desse hospital.

\section{Material e métodos}

Foram analisadas no laboratório de patologia clínica do HGF, no período de janeiro a dezembro de 2002, 662 amostras (aspirado traqueal, cateter, sangue e urina) de pacientes da UTI desse hospital. Dessas amostras, 259 continham microrganismos.

$\mathrm{O}$ isolamento das bactérias nas amostras foi realizado com meios de cultura específicos para cada uma delas, entre eles:

- caldo tripticaseína de soja TSB (Difco $\left.{ }^{\circledR}\right)$ : sangue;

- CLED (Difco ${ }^{\circledR}$ ): urina;

- ágar sangue e ágar MacConkey (Difco ${ }^{\circledR}$ ): aspirado traqueal e cateter ${ }^{(10)}$.

$\mathrm{O}$ isolamento de microrganismos no cateter foi realizado com cultura quantitativa, em que o cateter $( \pm 10 \mathrm{~cm})$ foi rolado nas placas de ágar sangue e ágar MacConkey (Di$\left.\mathrm{fco}^{\circledast}\right)$. As placas foram incubadas a $35^{\circ} \mathrm{C}$ em $5 \%$ de $\mathrm{CO}_{2}$ por 24 horas. Foram considerados positivos resultados acima de

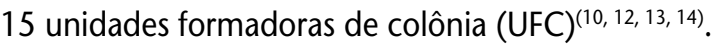

A urina coletada em cateteres foi semeada no CLED e foi realizada a cultura quantitativa utilizando alça calibrada 1:1.000, sendo as amostras incubadas em estufa a $35-37^{\circ} \mathrm{C}$

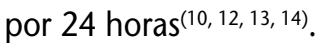

$\mathrm{O}$ aspirado traqueal foi semeado em ágar sangue e em ágar MacConkey, por meio de cultura semiquantitativa, na qual se utiliza solução de acetilcisteína diluída (1:4). As placas de ágar sangue foram incubadas em jarra a $35^{\circ} \mathrm{C}$ com $5 \%$ de $\mathrm{CO}_{2}$, por 24 horas, e as outras placas foram incubadas na estufa a $35^{\circ} \mathrm{C}$ também por 24 horas ${ }^{(10,12,13,14)}$.

O sangue foi coletado e diretamente colocado em garrafas (BACTEC), as quais foram mantidas na temperatura ambiente até serem incubadas no BACTEC 9240, 
um sistema automatizado de detecção fluorescente (nãoinvasivo) utilizado para monitoramento contínuo e simultâneo de amostras de sangue, a fim de detectar bactérias e fungos ${ }^{(10)}$.

A identificação e a sensibilidade a antibióticos foram feitas pelo sistema de automação MicroScan, utilizando painéis NUC2, NC21 e PUC21, convencionais liofilizados MicroScan e liofilizados MIC/Combo, destinados à determinação da suscetibilidade a agentes antimicrobianos ${ }^{(10)}$.

Os antimicrobianos utilizados no painel MicroScan PosCombo foram amoxicilina/ácido clavulânico (AMX/AC), cefotaxima (CFTX), ciprofloxacino (CIP), clindamicina (CLN), eritromicina (ERT), gentamicina (GEN), imipenem (IMP), levofloxacino (LVF), oxacilina (OXA), rifampicina (RIF), tetraciclina (TET), sulfametoxazol/trimetoprima (SFX/TMP), vancomicina (VAN), amicacina (AMI), amoxicilina/ácido clavulânico (AMX/AC), ampicilina/sulbactam (AMP/SUB), cefepima (CFP), ceftazidima (CFZ), ceftriaxona (CFX), cefuroxima (CFXO), nitrofurantoína (NTF), norfloxacino (NFL), ofloxacino (OFX), piperacilina (PIP), piperacilina/tazobactam (PIP/TAZ), ticarcilina/ácido clavulânico (TIC/AC), tobramicina $(\mathrm{TBR})^{(10)}$.

\section{Resultados}

De janeiro a dezembro de 2002, de 662 amostras, foram isolados 259 microrganismos (39,1\%). Entre as amostras que apresentaram maior positividade, estão:

- aspirado traqueal (88/259);

- sangue (77/259);

- urina (67/259);

- cateter venoso $(27 / 259)$.
Os principais microrganismos isolados do aspirado traqueal foram Pseudomonas aeruginosa (16\%), Klebsiella pneumoniae (15\%) e Acinetobacter baumannii (10\%). Os microrganismos isolados em cateter foram Staphylococcus coagulase negativa (SCN) (25\%), Acinetobacter baumannii (25\%), Staphylococcus aureus (24\%) e Klebsiella pneumoniae (11\%). Os microrganismos isolados em amostras de urina foram leveduras (44\%) e Klebsiella pneumoniae (16\%). Já os microrganismos isolados do sangue foram SCN (41\%), Staphylococcus aureus (17\%) e Pseudomonas aeruginosa (15\%).

As Tabelas 1, 2 e 3 mostram os percentuais de suscetibilidade de P. aeruginosa, K. pneumoniae e A. baumannii, respectivamente, isoladas nos vários materiais clínicos dos pacientes atendidos na UTI do HGF. Pode-se destacar na Tabela 1 que no aspirado traqueal, com isolamento de $P$. aeruginosa, os percentuais foram $40 \%$ de sensibilidade para aminoglicosídeos (GEN e AMI) e CIP; 60\% de sensibilidade para IMP; e $80 \%$ de sensibilidade para PIP/TAZ e TIC/AC. As mesmas cepas apresentaram resistência à CFX e à CFTX. No sangue, quase todos antibacterianos testados apresentaram $100 \%$ de suscetibilidade, exceto a GEN (60\%) e a CFTX (60\%).

$\mathrm{Na}$ Tabela 2 as cepas de K. pneumoniae no aspirado traqueal se destacam por $100 \%$ de suscetibilidade para IMP; $84 \%$ de suscetibilidade para CIP; $69 \%$ de suscetibilidade para AMI; e 54\% para CFZ. Na urina, 100\% apresentaram suscetibilidade para IMP e AMI; e 55\% para CIP e CFZ. já no cateter, $100 \%$ foram suscetíveis ao IMP e à AMI; $66 \%$ ao CIP e $33 \%$ à CFZ.

De acordo com a Tabela 3, para A. baumannii no aspirado traqueal destaca-se $89 \%$ de suscetibilidade para IMP; $56 \%$ de suscetibilidade para TIC/AC e AMP/SUB; e 100\% de resistência à TBR e à GEN. No cateter, 71\% foram suscetíveis ao IMP, à TIC/AC, à TBR e à GEN; e 15\% à AMP/SUB.

Tabela 1

Percentual de suscetibilidade de Pseudomonas aeruginosa de acordo com 0 material clínico de

\begin{tabular}{lcccccccc}
\hline Material clínico & $\mathbb{N}(\%)$ & $\mathbb{N}(\%)$ & $\mathbb{N}(\%)$ & $\mathbb{N}(\%)$ & $\mathbb{N}(\%)$ & $\mathbb{N}(\%)$ & $\mathbb{N}(\%)$ & $\begin{array}{c}N(\%) \\
\text { CFX }\end{array}$ \\
& PIP/TAZ & TIC/AC & CIP & IMP & GEN & AMI & CFT \\
Aspirado tra- & $12(80)$ & $12(80)$ & $6(40)$ & $9(60)$ & $6(40)$ & $6(40)$ & 0 & 0 \\
queal & & & & & & & & \\
Sangue & $12(100)$ & $12(100)$ & $12(100)$ & $12(100)$ & $7(60)$ & $12(100)$ & $12(100)$ & $7(60)$ \\
Cateter & - & - & - & - & - & - & - & - \\
Urina & - & - & - & - & - & - & - & - \\
Total & 24 & 24 & 18 & 21 & 13 & 18 & 12 & 7 \\
\hline
\end{tabular}

UTI: unidade de terapia intensiva; HGF: Hospital Geral de Fortaleza; PIP/TAZ: piperacilina/tazobactam; TIC/AC: ticarcilina/ácido clavulânico; CIP: ciprofloxacino; IMP: imipenem; CEN: gentamicina; AMI: amicacina; CFX: ceftriaxona; CFTX: cefotaxima. 


\section{Tabela 2}

Material clínico

Aspirado traqueal

Sangue

Cateter

Urina

Total

\section{Percentual de suscetibilidade de Klebsiella pneumoniae de acordo com 0 material clínico de} pacientes da UTI do HGF

UTI: unidade de terapia intensiva; HCF: Hospital Geral de Fortaleza; CFZ: ceftazidima; IMP: imipenem; CIP: ciprofloxacino; AMI: amicacina.

\section{$N(\%)$}

CFZ

7 (54)

$1(33)$

$6(55)$

14

$N(\%)$

IMP

$13(100)$

$3(100)$

$11(100)$

27
$\mathbb{N}(\%)$

CIP

$11(84)$

$2(66)$

$6(55)$

19
$\mathrm{N}(\%)$

AMI

$9(69)$

$11(100)$

23

\section{Tabela 3 pacientes da UTI do HGF}

\begin{tabular}{|c|c|c|c|c|c|}
\hline Material clínico & $\mathrm{N}(\%)$ IMP & N (\%) TIC-AC & N (\%) AMP/SUB & $\mathrm{N}(\%) \mathrm{TBR}$ & N (\%) GEN \\
\hline $\begin{array}{l}\text { Aspirado tra- } \\
\text { queal }\end{array}$ & $8(89)$ & $5(56)$ & $5(56)$ & 0 & 0 \\
\hline Sangue & - & - & - & - & - \\
\hline Cateter & $5(71)$ & $5(71)$ & $1(15)$ & $5(71)$ & $5(71)$ \\
\hline Urina & - & - & - & - & - \\
\hline Total & 13 & 10 & 6 & 5 & 5 \\
\hline
\end{tabular}

UTI: unidade de terapia intensiva; HGF: Hospital Geral de Fortaleza; IMP: imipenem; TIC/AC: ticarcilina/ácido clavulânico; AMP/SUB: ampicilina/sulbactam; TBR: tobramicina; GEN: gentamicina.

A Figura 1 mostra o percentual de suscetibilidade de S. aureus em cateteres de pacientes atendidos na UTI do HGF, em que se pode destacar $100 \%$ de suscetibilidade para VAN; $75 \%$ para RIF; e apenas $25 \%$ de suscetibilidade para OXA, GEN e IMP, respectivamente.
Na Figura 2 pode ser observado para SCN 100\% de suscetibilidade para VAN; $75 \%$ de suscetibilidade para RIF; e $7 \%$ de suscetibilidade para OXA. Para S. aureus pode se destacar $100 \%$ de suscetibilidade para VAN e RIF; e 50\% de suscetibilidade para OXA.

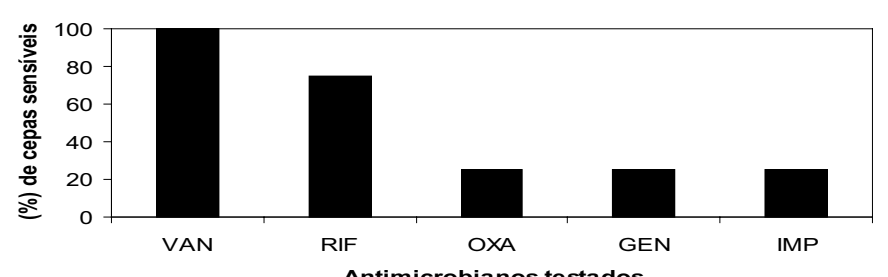

Figura 1 - Percentual de suscetibilidade aos antimicrobianos das cepas de Staphylococcus aureus isoladas de cateteres de pacientes da UTI do Hospital Geral de Fortaleza (HGF)

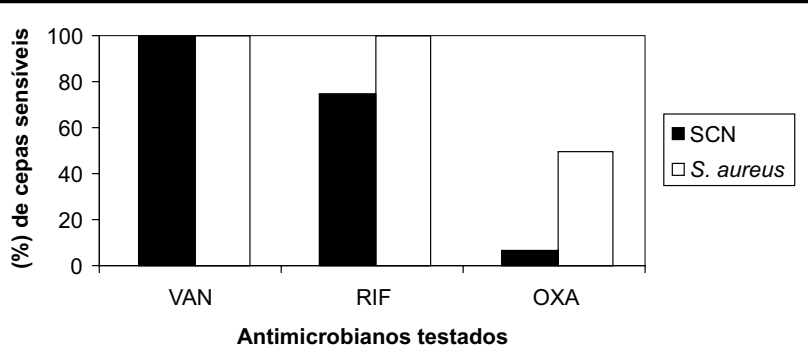

Figura 2 - Percentual de suscetibilidade aos antimicrobianos das cepas de Staphylococcus coagulase negativa (SCN) e Staphylococcus aureus isoladas do sangue de pacientes da UTI do Hospital Geral de Fortaleza (HCF) 


\section{Discussão}

Embora as UTIs representem apenas 5\% a 10\% dos leitos de um hospital, estima-se que nelas ocorram cerca de $25 \%$ das infecções hospitalares ${ }^{(5,26)}$. A média das taxas de infecção hospitalar em 196 UTIs americanas que participaram do programa National Nosocomial Infections Surveillance System/Center for Disease Control and Prevention (NNISS/ $\mathrm{CDC})^{(9,23)}$ está próxima de $9,2 \%$ (com densidade de infecção de 23,7 infecções por mil pacientes/dia), o que equivale a uma taxa duas a cinco vezes maior do que as encontradas em enfermarias ${ }^{(2)}$. Na UTI do HGF verificamos a taxa média de infecção hospitalar de 13,4 infecções hospitalares por mil pacientes/dia, sendo menor do que a do NNISS.

Um estudo europeu de prevalência de infecções hospitalares em UTIs (excluídas as unidades coronarianas e as UTIs infantis e especiais) merece destaque por ter contado com a participação de 17 países, 1.417 UTIs e 10.038 pacientes acompanhados ${ }^{(20,23)}$. Neste trabalho foram detectados 4.501 (44,8\%) pacientes infectados, dos quais $2.064(20,6 \%)$ eram portadores de infecção hospitalar ${ }^{(7)}$. Em nosso meio o HGF, em casuística com metodologia NNISS, apresenta taxa de infecções hospitalares de 4,7\% (com densidade de infecção de 13,4 por mil pacientes/dia).

Os patógenos nosocomiais são selecionados por causa de mudanças nas práticas médicas e devido ao uso de antimicrobianos(2, 11, 24).

Klebsiella pneumoniae, como outros membros da família das enterobactérias, é um patógeno oportunista que se manifesta em proporções significativas nas infecções hospitalares, como infecções do trato urinário, pneumonias, septicemias e infecções de tecidos moles ${ }^{(11)}$. Em nosso estudo foi detectada a presença de $K$. pneumoniae em todas as amostras estudadas.

Segundo Stamm e Coutinho(19), ao estudarem infecções urinárias relacionadas ao cateter urinário observaram que a freqüência maior era $E$. coli, vindo em seguida Enterobacter sp. SCN e S. aureus, e as leveduras em quinto lugar, de acordo com freqüência microbiana. No nosso trabalho, as leveduras foram as mais isoladas.

Em um estudo realizado por Menezes et al. ${ }^{(13)}$ sobre infecções urinárias em pacientes cateterizados em um hospital de Fortaleza, ficou constatado que as bactérias mais prevalentes foram K. pneumoniae, P. aeruginosa e E. coli. Essas bactérias continuam sendo importante causa de infecções urinárias em pacientes do HGF, conforme descrito em nosso estudo.

Em estudo epidemiológico realizado por Wang e Chang ${ }^{(25)}$, os microrganismos gram-negativos mais co- mumente isolados em UTI foram P. aeruginosa, E. coli, Klebsiella sp. e Acinetobacter sp. Os dados coincidem com os encontrados em nosso estudo, confirmando a importância mundial desses patógenos. No mesmo estudo, o antimicrobiano com maior atividade foi o IMP. Esse também foi o resultado encontrado em nosso trabalho.

A Pseudomonas aeruginosa, o patógeno mais comum envolvido em infecções hospitalares, é causadora de infecções oportunistas em pacientes imunocomprometidos, além de ser comum em UTIs ${ }^{(10,16,22)}$.

Nathens et al. ${ }^{(17)}$ observaram que, apesar da freqüência de infecções por agentes de baixa patogenicidade, as infecções hospitalares resultam em maior letalidade nos pacientes críticos. Por outro lado, Fagon et al. ${ }^{(6)}$, utilizando modelos matemáticos, concluíram que as infecções hospitalares aumentam o risco de morte do paciente atendido na UTI, sendo esse efeito altamente provável para pneumonia, duvidoso para bacteremia e incerto para infecção urinária. Esse risco aumenta o tempo de permanência na UTI ${ }^{(7)}$.

Em relação ao percentual de suscetibilidade das cepas bacterianas isoladas, observamos que as $P$. aeruginos $a$ isoladas nos aspirados traqueais apresentaram total sensibilidade à PIP e $100 \%$ de resistência à CFX e à CFTX. Isoladas da urina, apresentaram suscetibilidade apenas à PIP, sendo resistentes a todos os antimicrobianos testados. As K. pneumoniae isoladas de aspirados traqueais foram totalmente sensíveis ao IMP; não mostraram resistência total a nenhum antimicrobiano testado; apresentaram suscetibilidade de 54\% à CFZ; e no cateter elas apresentaram alta resistência.

Menezes et al.(15) observaram, ao estudarem o percentual de infecção e resistência de bacilos gram-negativos não-fermentadores em um hospital do Ceará, que o microrganismo mais comumente isolado foi a $P$. aeruginosa. $O$ setor hospitalar com maior índice de amostras positivas foi a UTI, sendo o aspirado traqueal a amostra biológica com maior índice de positividade. Dos antimicrobianos testados, os mais potentes foram IMP e CIP. Os antimicrobianos menos potentes foram as cefalosporinas e a AMP, o que está de acordo com os dados apresentados em nosso estudo.

Em um estudo do percentual de sensibilidade a antimicrobianos, realizado no HGF em 2001, o microrganismo mais comumente isolado foi a $P$. aeruginosa proveniente da UTI e isolada de amostras de urina e de aspirado traqueal. As cepas mostraram sensibilidade às penicilinas anti-Pseudomonas combinadas com inibidores de betalactamases $\mathrm{e}$ apresentaram resistência às cefalosporinas, enquanto o IMP apresentou boa atividade. Novamente, os dados anteriores confirmam nosso estudo ${ }^{(14)}$. 
O S. aureus e o SCN foram isolados principalmente do cateter, sendo os dois suscetíveis à VAN (100\%). Por outro lado, os Staphylococcus vêm mostrando elevado índice de resistência à OXA no meio hospitalar.

Relatos de hospitais em diferentes regiões brasileiras encontraram $30 \%$ a $100 \%$ de S. aureus resistentes à OXA. Essa resistência à OXA varia conforme a região ou mesmo de acordo com o hospital analisado, devendo ser avaliada localmente ${ }^{(1)}$. Em nosso trabalho, observamos uma sensibilidade de $7 \%$ à OXA entre cepas SCN.

Em estudo de caracterização bacteriana em culturas de ponta de cateter, realizado no HGF em 2000, ficou evidenciado que os microrganismos mais prevalentes foram SCN, $P$. aeruginosa e K. pneumoniae. Quanto ao percentual de sensibilidade, os SCN mostraram-se totalmente sensíveis à VAN e resistentes a algumas penicilinas, entre elas a OXA. A $P$. aeruginosa mostrou ser resistente a grande parte dos antimicrobianos testados. Os resultados coincidem, em parte, com os apresentados neste estudo ${ }^{(12)}$.

A resistência das diversas espécies bacterianas aos antimicrobianos é extremamente variável entre países, regiões e origem hospitalar e/ou comunitária das estirpes. Algumas espécies apresentam resistência amplamente difundida em todo o mundo, como no caso do S. aureus(21).

Entre os microrganismos que sofreram grandes modificações na sensibilidade aos antimicrobianos com o correr dos anos, destacam-se: estafilococos, enterobactérias,
P. aeruginosa, A. baumannii e, mais recentemente, hemófilos, gonococos, enterococos e pneumococos. Na atualidade, é motivo de grande preocupação entre microbiologistas e médicos a resistência entre as bactérias gram-positivas, que vêm se tornando bactérias-problema na terapêutica antiinfecciosa(21).

\section{Conclusão}

- As bactérias gram-positivas e gram-negativas estão envolvidas em infecções hospitalares na UTI do HGF.

- Klebsiella pneumoniae e Pseudomonas aeruginosa são os principais bacilos gram-negativos envolvidos em infecções na UTI do HGF.

- O Acinetobacter baumannii começa a se configurar como importante patógeno nas infecções que ocorrem na UTI do HGF.

- Os cocos gram-positivos responsáveis por infecções na UTI do HGF são Staphylococcus coagulase negativa e Staphylococcus aureus.

- As cefalosporinas de terceira geração não representam uma alternativa terapêutica muito eficiente no tratamento de infecções que ocorrem na UTI do HGF.

- O imipenem, as penicilinas anti-Pseudomonas combinadas a inibidores de betalactamases e o ciprofloxacino representam alternativas terapêuticas no tratamento de infecções provocadas por esses patógenos.

\section{Referências}

I. AMYES, S.G.B.; GEMELL, C.G. Antibiotic resistance. J. Med. Microbiol, v. 46, p. 436-70, 1997.

2. ASSOCIATION FOR PROFESSIONALS IN INFECTION CONTROLAND EPIDEMIOLOGY (APIC). Infection control and applied epidemilogy principles and pratice. IN: RUSSEL, N. Olmsted: Mosby Year Book, 1996. p. 173-86.

3. BOLICK, D. et al. Segurança e controle de infecção. I ed. Rio de Janeiro: Reichmann \& Affonso Editores, 2000.

4. COUTO, R.C.; PEDROSA, T.M.G.; NOGUEIRA, J.M. Infecção hospitalar: epidemiologia e controle. 2 ed. Belo Horizonte: Ed. Medsi, 1999.

5. FARR, B.M. Infection control in intensive care units: modern problems modern solutions. Curr Opinion Infect Dis, v. 32, p. p. 520-5, 1993.

6. FAGON, J.l. et al. Mortality attributable to nosocomial infections in the ICU. Infect Control Hosp Epidemiol, v. I5, p. 428-34, 1994.

7. FERNANDES, A.T.; FERNANDES, M.O.V.; FILHO, N.R. Infecção hospitalar e suas interfaces na área da Saúde. São Paulo: Ed. Atheneu, 2000

8. GOMES, M.J.V.M.; REIS, A.M.M. Ciências farmacêuticas: uma abordagem em farmácia hospitalar. I ed. São Paulo. Ed. Medsi, 200I.

9. JARVIS, W.R. et al. Nosocomial infection rates in adult and pediatric intensive care units in the United States. Am J Med, v. 91, n. 3B, p. |85-9|, 1992.

10. KONEMAN, E.W. et al. A função do laboratório de microbiologia no diagnóstico de doenças infecciosas: indicações para prática e manejo. In: KONEMAN, E.W. et al. Diagnóstico microbiológico texto e atlas colorido. 5 ed. São Paulo: Medsi, 200 I. cap 2. p. 69-176.

I I. LOPES, A C.S.; RODRIGUES, J.F.; MARAIS JR., M.A. Molecular typing of Klebsiella pneumoniae isolates from public hospitals in Recife, Brazil. Microbiol Research, v. 160, p. 37-46, 2005.

12. MENEZES, E.A. et al. Caracterização bacteriana em culturas de 
ponta de cateter no Hospital Geral de Fortaleza. Rev Bras Anal Clin, v. 34, n. 3, p. I 5 -4, 2002.

13. MENEZES, E.A. et al. Infecções urinárias por Pseudomonas aeruginosa em pacientes cateterizados na clínica urológica da Santa Casa de Misericórdia de Fortaleza - Ceará. Rev. Bras Anal Clin, v. 35, n. 2, p. 77-9, 2003 a.

14. MENEZES, E.A. et al. Perfil de resistência aos antimicrobianos de Pseudomonas isoladas no Hospital Geral de Fortaleza. Rev Bras Anal Clin, v. 35, n. 4, p. 177-80, 2003 b.

15. MENEZES, E.A. et al. Perfil de infecção e resistência aos antimicrobianos de bacilos gram-negativos não fermentadores isolados no Laboratório de Patologia Clínica Dr. Edíson Gurgel da Santa Casa de Misericórdia de Fortaleza-CE. Rev Bras Anal Clin, v. 36, n. 4, p. 209-12, 2004.

16. MOOLENAAR, R.L. et al. A prolonged outbreak of Pseudomonas aeruginosa in a neonatal intensive care unit: did staff fingernails play a role in disease transmission? Infect Control Hosp Epidemiol, v. 21, p. 80-5, 2000.

17. NATHENS, A.B. et al. Nosocomial infection in the surgical ICU: attributable morbidity and mortality. Abstract 192. Critical Care Med, v. 24, n. A96, 1996.

18. NETO, V.A.; LEVI, G.C.; LOPES, H.V. Antibiótico na prática médica. 5 ed. São Paulo: Editora Roca, 2000.

19. STAMM, A.M.N.F; COUTINHO, M.S.S.A. Infecção do trato urinário relacionada ao cateter vesical de demora: incidência e fatores de risco, Florianópolis-SC. Rev Assoc Méd Bras, v. 21, p. 123-6, 1999.

20. SPENCER, R.C. Epidemiology of infection in ICUs. Intensive Care Med, v. 20, n. 5, p. 2-6, 1994

21. TAVARES, W. Bactérias gram-positivas problemas: resistência do estafilococo, do enterococo e do pneumococo aos antimicrobianos. Rev Soc Bras Med Trop, v. 33, n. 3, p. 281 301,2000

22.TSAKRIS,A et al. Outbreak of infections caused by Pseudomonas aeruginosa producingVIM-I carbapenemase in Greece.J Clin Microbiol, v. 38, p. I290-2, 2000.

23. VICENT, J.L. et al. The prevalence of nosocomial infection in intensive care units in Europe: results of the European prevalence of infection in intensive care (EPIC) study. EPIC Internetional Advisory Committee. JAMA, v. 274, n. 8, p. 639-44, 1995

24. ZANON, U.; NEVES, J. Infecções hospitalares: prevenção, diagnóstico e tratamento. Rio de Janeiro: Ed. Medsi, 1987.

25. WANG, H.; CHANG, M. Surveillance for antimicrobial resistance among clinical isolates of gram-negative bacteria from intensive care unit patients in China, 1996 to 2002. Diagnostic Microbiol Infect Dis, v. 5I, n. I234-4I, 2004.

26. WINDMER, A.F. Infection control and prevention strategies in the ICU. Intensive Care Med, v. 20, n. 5, p. 7-II, 1994. 